Pacific

Journal of

Mathematics

\title{
GENERALIZED COMPLEX SUBMANIFOLDS
}

JAMES BARTON AND MATHIEU STIÉNON 


\title{
GENERALIZED COMPLEX SUBMANIFOLDS
}

\author{
JAMES BARTON AND MATHIEU STIÉNON
}

\begin{abstract}
We introduce the notion of twisted generalized complex submanifolds and describe an equivalent characterization in terms of Poisson-Dirac submanifolds. Our characterization recovers a result of Vaisman (2007). An equivalent characterization is also given in terms of spinors. As a consequence, we show that the fixed locus of an involution preserving a twisted generalized complex structure is a twisted generalized complex submanifold. We also prove that a twisted generalized complex manifold has a natural Poisson structure. We also discuss generalized Kähler submanifolds.
\end{abstract}

\section{Introduction}

Throughout this paper $M$ will denote a smooth manifold. Generalized complex structures were originally defined by Hitchin [2003], and further studied by Gualtieri [2003]. Examples of generalized complex structures include symplectic and complex manifolds. In order to define generalized complex structures we will recall some structures on $T M \oplus T^{*} M$. The Courant bracket was defined in [Courant 1990] as

$$
\llbracket X+\xi, Y+\eta \rrbracket=[X, Y]+\mathscr{L}_{X} \eta-\mathscr{L}_{Y} \xi-\frac{1}{2} d(\eta(X)-\xi(Y))
$$

for all $X, Y \in \mathfrak{X}(M)$ and $\xi, \eta \in \Omega(M)$. There also exist smoothly varying nondegenerate symmetric bilinear forms on each fibre of $T M \oplus T^{*} M$. These are defined as

$$
\langle X+\xi, Y+\eta\rangle=\frac{1}{2}(\xi(Y)+\eta(X))
$$

for all $X, Y \in T_{m} M, \xi, \eta \in T_{m}^{*} M$ and $m \in M$.

A generalized complex structure is a smooth map $J: T M \oplus T^{*} M \rightarrow T M \oplus T^{*} M$ such that $J^{2}=-\mathrm{Id}, J J^{*}=\mathrm{Id}$, and the $+i$-eigenbundle of $J$ is involutive with respect to the Courant bracket.

The primary objects of study in this paper are twisted manifolds. A manifold $M$ endowed with a closed 3-form $\Omega$ will be called twisted. A twisted manifold has

MSC2000: 53C56, 53D17, 53D35.

Keywords: generalized complex geometry, Poisson bivector, Poisson-Dirac submanifold.

Research partially supported by NSF grant DMS03-06665. Stiénon is an ESI Junior Research Fellow. 
another well known bracket on $\mathfrak{X}(M) \oplus \Omega(M)$ : the twisted Courant bracket. This bracket was defined in [Ševera and Weinstein 2001] as

$$
\llbracket X+\xi, Y+\eta \rrbracket_{\Omega}=[X, Y]+\mathscr{L}_{X} \eta-\mathscr{L}_{Y} \xi-\frac{1}{2} d(\eta(X)-\xi(Y))+i_{Y} i_{X} \Omega
$$

A twisted generalized complex structure is a smooth map $J: T M \oplus T^{*} M \rightarrow$ $T M \oplus T^{*} M$ such that $J^{2}=-\mathrm{Id}, J J^{*}=\mathrm{Id}$ and the $+i$-eigenbundle of $J$ is involutive with respect to (1-2), rather than (1-1). The triple $(M, \Omega, J)$ will be called a twisted generalized complex manifold. Indeed, generalized complex manifolds can be defined for arbitrary Courant algebroids. See [Barton 2007] for details.

The aim of this work is to characterize when a submanifold of a twisted generalized complex manifold is also a twisted generalized complex manifold. In the untwisted case, several notions of generalized complex submanifolds have been recently introduced. The notion defined here is similar to the one in [Ben-Bassat and Boyarchenko 2004] and [Vaisman 2007]. A different notion of generalized complex submanifolds appears in [Gualtieri 2003].

Definition 1.1. A twisted immersion, from one twisted manifold $(N, \Upsilon)$ to another $(M, \Omega)$, is defined as a smooth immersion $h: N \rightarrow M$ with $\Upsilon=h^{*} \Omega$. A twisted generalized complex immersion from $\left(N, \Upsilon, J^{\prime}\right)$ to $(M, \Omega, J)$ is a twisted immersion $h:(N, \Upsilon) \rightarrow(M, \Omega)$ such that the pullback of the $+i$-eigenbundle of $J$ is the $+i$-eigenbundle of $J^{\prime}$. In this case, $N$ is called a twisted generalized complex submanifold of $M$.

By splitting vectors and covectors, a twisted generalized complex structure can be written as

$$
J=\left(\begin{array}{cc}
\phi & \pi^{\sharp} \\
\sigma_{b} & -\phi^{*}
\end{array}\right) .
$$

Here $\phi$ is an endomorphism of $T M, \pi^{\sharp}: T^{*} M \rightarrow T M$ is the bundle map induced by a bivector field $\pi$, and $\sigma_{b}: T M \rightarrow T^{*} M$ is the bundle map induced by a 2 -form $\sigma$. The fact that $J^{2}=-$ Id also leads to the following formulas:

$$
\phi^{2}+\pi^{\sharp} \sigma_{b}=-\mathrm{Id}, \quad \phi \pi^{\sharp}=\pi^{\sharp} \phi^{*}, \quad \text { and } \quad \phi^{*} \sigma_{b}=\sigma_{b} \phi .
$$

These facts, and others, were first noted in [Crainic 2004]. These results were also described using Poisson quasi-Nijenhuis manifolds in [Stiénon and Xu 2007]. The conditions for a submanifold to be twisted generalized complex will be expressed in terms of this splitting. Using the theory of Lie bialgebroids we also show that $\pi$ from (1-3) is a Poisson bivector field, which, for the untwisted case, is a standard result [Abouzaid and Boyarchenko 2006; Crainic 2004]. 
Our work was inspired by [Stiénon and Xu 2008], where the reduction of generalized complex structures is studied. The main result was also independently obtained by Vaisman [2007].

This paper is organized as follows. In Section 2, we recall some of the basic facts of Dirac structures. In particular we describe the pull back. In Section 3, we prove that a twisted generalized complex manifold carries a natural Poisson structure. In Section 4, we define the induced generalized complex structure, and characterize when it has the required properties. In Section 5, we prove the main theorem of this paper, and provide examples. Twisted generalized complex involutions are also introduced in this section. In Section 6, we determine when a submanifold of a holomorphic Poisson manifold is itself endowed with an induced holomorphic Poisson structure. Section 7 is a restatement of our main result in terms of spinors. The last section discusses generalized Kähler submanifolds.

\section{Dirac structures}

The aim of this section is to recall Dirac structures, and their pull backs. Before considering bundles, we will consider a vector space $V$. In this case a Dirac structure is nothing more than a maximal isotropic subspace of $V \oplus V^{*}$. Let $q_{1}$ denote the projection of $V \oplus V^{*}$ onto $V$, and $q_{2}$ the projection onto $V^{*}$.

If $L$ is a Dirac structure then there exists a natural skew-symmetric bilinear form $\Lambda$ on $L$ defined by

$$
\Lambda(X+\xi, Y+\eta)=\xi(Y)=-\eta(X) \text { for all } X+\xi, Y+\eta \in L .
$$

It is easy to see that

$$
\Lambda\left(X+\xi_{1}, Y+\eta_{1}\right)=\Lambda\left(X+\xi_{2}, Y+\eta_{2}\right) \text { for all } X+\xi_{1,2}, Y+\eta_{1,2} \in L,
$$

and

$$
\Lambda\left(X_{1}+\xi, Y_{1}+\eta\right)=\Lambda\left(X_{2}+\xi, Y_{2}+\eta\right) \text { for all } X_{1,2}+\xi, Y_{1,2}+\eta \in L .
$$

Hence, there exists a 2-form $\varepsilon$ on $q_{1}(L)$ defined by

$$
\varepsilon(X, Y)=\Lambda(X+\xi, Y+\eta) \text { for all } X+\xi, Y+\eta \in L,
$$

and a 2 -form $\theta$ on $q_{2}(L)$ defined by

$$
\theta(\xi, \eta)=-\Lambda(X+\xi, Y+\eta) \text { for all } X+\xi, Y+\eta \in L .
$$

If $X \in q_{1}(L)$ then there exists some $\xi \in V^{*}$ with $X+\xi \in L$; furthermore $\varepsilon(X, Y)=\xi(Y)$ for all $Y \in q_{1}(L)$. Thus $i_{X} \varepsilon=\left.\xi\right|_{q_{1}(L)}$, and

$$
X+\xi \in L \quad \Longleftrightarrow \quad X \in q_{1}(L) \text { and } i_{X} \varepsilon=\left.\xi\right|_{q_{1}(L)} .
$$


Thus knowing the Dirac structure $L$ is exactly the same as knowing the subspace $q_{1}(L)$ and the 2-form $\varepsilon$. Similarly, $L$ is equivalent to the pair $\left(q_{2}(L), \theta\right)$. Thus any subspace $R \subseteq V$ endowed with a 2-form $\varepsilon$ on $R$ defines a Dirac structure $L(R, \varepsilon)$ :

$$
L(R, \varepsilon)=\left\{X+\xi \in R \oplus V^{*}: i_{X} \varepsilon=\left.\xi\right|_{R}\right\},
$$

and any subspace $S \subseteq V^{*}$ endowed with a 2-form $\pi$ on $S$ defines a Dirac structure $L(S, \theta)$ :

$$
L(S, \theta)=\{X+\xi \in V \oplus S: \theta(\xi, \eta)=-\eta(X) \text { for all } \eta \in S\} .
$$

Details of these constructions can be found in [Courant 1990]. Let $W$ be another vector space and $\varphi: V \rightarrow W$ a linear map. The map $\varphi$ can be used to both pull Dirac structures back from $W$ to $V$, and push Dirac structures forward from $V$ to $W$. Let $(R, \varepsilon)$ be a Dirac structure on $W$, with $R \subseteq W$ and $\varepsilon \in \Gamma\left(\bigwedge^{2} R^{*}\right)$. A Dirac structure on $V$ is defined by $\left(\varphi^{-1} R, \varphi^{*} \varepsilon\right)$. This Dirac structure is called the pull back of $(R, \varepsilon)$ under $\varphi$. Similarly if $(S, \theta)$ is a Dirac structure on $V$, with $S \subseteq V^{*}$ and $\theta$ defined on $S$, then $\left(\left(\varphi^{*}\right)^{-1} S, \varphi_{*} \theta\right)$ defines a Dirac structure on $W$. This Dirac structure is called the push forward of $(S, \theta)$ under $\varphi$. These two maps of Dirac structures are denoted by $\mathscr{F}_{\varphi}$ and $\mathscr{B}_{\varphi}$. It is very easy to see that for a Dirac structure $L$ on $W$

$$
B_{\varphi}(L)=\left\{X+\varphi^{*} \xi: X \in V, \xi \in W^{*} \text { such that } \varphi X+\xi \in L^{\prime}\right\}
$$

and for a Dirac structure $L^{\prime}$ on $V$

$$
\mathscr{F}_{\varphi}\left(L^{\prime}\right)=\left\{\varphi X+\xi: X \in V, \xi \in W^{*} \text { such that } X+\varphi^{*} \xi \in L\right\} .
$$

Dirac structures can also be defined for a twisted manifold $(M, \Omega)$. A Dirac structure is a smooth subbundle $L \subseteq T M \oplus T^{*} M$ for which each fibre is a Dirac structure of the corresponding fibre of $T M \oplus T^{*} M$, and whose space of sections is closed under the twisted Courant bracket (1-2). The restriction of the twisted Courant bracket to a Dirac structure is a Lie bracket; thus a Dirac structure is naturally a Lie algebroid.

The definitions of push forward and pull back can be reformulated for Dirac structures on manifolds. We will only consider the pull back of a Dirac structure, but more on the push forward can be found in [Bursztyn and Radko 2003] and [Stiénon and Xu 2008]. We note that the pull back of a Dirac structure is automatically a maximal isotropic, but it need not be smooth or involutive.

The last lemma of this section will be used to characterize when the pullback bundle is involutive. Let $(M, \Omega)$ and $(N, \Upsilon)$ be two twisted manifolds with an immersion $\varphi: N \rightarrow M$. Two sections

$$
\sigma_{N}=X+\xi \in \mathfrak{X}(N) \oplus \Omega(N) \quad \text { and } \quad \sigma_{M}=Y+\eta \in \mathfrak{X}(M) \oplus \Omega(M)
$$


are said to be $\varphi$-related, denoted by $\sigma_{N} \stackrel{\varphi}{\sim} \sigma_{M}$, if $Y=\varphi_{*} X$ and $\xi=\varphi^{*} \eta$. The following lemma is an extension of [Stiénon and Xu 2008, Lemma 2.2]. This lemma is also true for complex sections, which is when it will be applied.

Lemma 2.1. Assume that $\sigma_{N}^{i} \in \Gamma\left(T N \oplus T^{*} N\right)$ and $\sigma_{M}^{i} \in \Gamma\left(T M \oplus T^{*} M\right)$ satisfy $\sigma_{N}^{i} \stackrel{\varphi}{\rightsquigarrow} \sigma_{M}^{i}$, for $i=1,2$. Then, if $\varphi$ is a twisted immersion,

$$
\llbracket \sigma_{N}^{1}, \sigma_{N}^{2} \rrbracket_{\Upsilon} \stackrel{\varphi}{\rightsquigarrow} \llbracket \sigma_{M}^{1}, \sigma_{M}^{2} \rrbracket_{\Omega} .
$$

Proof. Write $\sigma_{N}^{i}=X^{i}+\xi^{i}$ and $\sigma_{M}^{i}=Y^{i}+\eta^{i}$, where $X^{i}+\xi^{i} \in \mathfrak{X}(N) \oplus \Omega(N)$ and $Y^{i}+\eta^{i} \in \mathfrak{X}(M) \oplus \Omega(M)$, for $i=1$, 2. Since $\sigma_{N}^{i} \stackrel{\varphi}{\rightsquigarrow} \sigma_{M}^{i}$, for $i=1,2$, then $\varphi_{*} X^{i}=Y^{i}$ and $\varphi^{*} \eta^{i}=\xi^{i}$. By definition

$$
\llbracket \sigma_{N}^{1}, \sigma_{N}^{2} \rrbracket_{\Upsilon}=\left[X^{1}, X^{2}\right]+\mathscr{L}_{X^{1}} \xi^{2}-\mathscr{L}_{X^{2}} \xi^{1}+\frac{1}{2} d\left(\xi^{1}\left(X^{2}\right)-\xi^{2}\left(X^{1}\right)\right)+i_{X^{2}} i_{X^{1}} \Upsilon,
$$

and

$$
\llbracket \sigma_{M}^{1}, \sigma_{M}^{2} \rrbracket_{\Omega}=\left[Y^{1}, Y^{2}\right]+\mathscr{L}_{Y^{1}} \eta^{2}-\mathscr{L}_{Y^{2}} \eta^{1}+\frac{1}{2} d\left(\eta^{1}\left(Y^{2}\right)-\eta^{2}\left(Y^{1}\right)\right)+i_{Y^{2}} i_{Y^{1}} \Omega .
$$

Now

$$
\varphi_{*}\left[X^{1}, X^{2}\right]=\left[\varphi_{*} X^{1}, \varphi_{*} X^{2}\right]=\left[Y^{1}, Y^{2}\right]
$$

and

$$
\begin{aligned}
\varphi^{*}\left(\mathscr{L}_{Y^{1}} \eta^{2}\right) & =\varphi^{*}\left(i_{Y^{1}} d \eta^{2}+d i_{Y^{1}} \eta^{2}\right)=\varphi^{*} i_{\varphi_{*} X^{1}} d \eta^{2}+\varphi^{*} d\left(\eta^{2}\left(Y^{1}\right)\right) \\
& =i_{X^{1}} \varphi^{*} d \eta^{2}+d\left(\xi^{2}\left(X^{1}\right)\right)=i_{X^{1}} d \xi^{2}+d i_{X^{1}} \xi^{2}=\mathscr{L}_{X^{1}} \xi^{2} .
\end{aligned}
$$

Similarly,

$$
\varphi^{*}\left(\mathscr{L}_{Y^{2}} \eta^{1}\right)=\mathscr{L}_{X^{2}} \xi^{1}
$$

The second last term becomes

$$
\varphi^{*} d\left(\eta^{1}\left(Y^{2}\right)-\eta^{2}\left(Y^{1}\right)\right)=d\left(\xi^{1}\left(X^{2}\right)-\xi^{2}\left(X^{1}\right)\right),
$$

since

$$
\varphi^{*}\left(\eta^{1}\left(Y^{2}\right)\right)=\varphi^{*}\left(\eta^{1}\left(\varphi_{*} X^{2}\right)\right)=\left(\varphi^{*} \eta^{1}\right)\left(X^{2}\right)=\xi^{1}\left(X^{2}\right) .
$$

Finally, because $\varphi$ is a twisted immersion, the following holds.

$$
\varphi^{*} i_{Y^{2}} i_{Y^{1}} \Upsilon=\varphi^{*} i_{\varphi_{*} X^{2}} i_{\varphi_{*} X^{1}} \Upsilon=i_{X^{2}} i_{X^{1}} \varphi^{*} \Upsilon=i_{X^{2}} i_{X^{1}} \Omega .
$$

\section{The Poisson bivector field associated to a generalized complex structure}

For the usual Courant bracket it is well known that the existence of a generalized complex structure leads to a Poisson bivector [Abouzaid and Boyarchenko 2006; Crainic 2004; Lindström et al. 2005; Hu 2005]. In this chapter we will obtain the same result for arbitrary Courant algebroids and also give a new way of expressing the Poisson bivector. 
A Courant algebroid [Liu et al. 1997] is a triple consisting of a vector bundle $E \rightarrow M$ equipped with a nondegenerate symmetric bilinear form $\langle\cdot, \cdot\rangle$, a skewsymmetric bracket $\llbracket \cdot, \cdot \rrbracket$ on $\Gamma(E)$, and a smooth bundle map $E \stackrel{\rho}{\rightarrow} T M$ called the anchor. These induce a natural differential operator $\mathscr{D}: C^{\infty}(M) \rightarrow \Gamma(E)$ defined by

$$
\langle\mathscr{D} f, a\rangle=\frac{1}{2} \rho(a) f,
$$

for all $f \in C^{\infty}(M)$ and $a \in \Gamma(E)$. These structures must obey the following formulas for all $a, b, c \in \Gamma(E)$ and $f, g \in C^{\infty}(M)$ :

$$
\begin{aligned}
\rho(\llbracket a, b \rrbracket) & =[\rho(a), \rho(b)], \\
\llbracket \llbracket a, b \rrbracket, c \rrbracket+\llbracket \llbracket b, c \rrbracket, a \rrbracket+\llbracket \llbracket c, a \rrbracket, b \rrbracket & =\frac{1}{3} \mathscr{D}(\langle\llbracket a, b \rrbracket, c\rangle+\langle\llbracket b, c \rrbracket, a\rangle+\langle\llbracket c, a \rrbracket, b\rangle), \\
\llbracket a, f b \rrbracket & =f \llbracket a, b \rrbracket+(\rho(a) f) b-\langle a, b\rangle \mathscr{D} f, \\
\rho \circ \mathscr{D} & =0, \text { that is, }\langle\mathscr{D} f, \mathscr{D} g\rangle=0, \\
\rho(a)\langle b, c\rangle & =\langle\llbracket a, b \rrbracket+\mathscr{D}\langle a, b\rangle, c\rangle+\langle b, \llbracket a, c \rrbracket+\mathscr{D}\langle a, c\rangle\rangle .
\end{aligned}
$$

The relation

$$
\llbracket \mathscr{D} f, a \rrbracket+\mathscr{D}\langle\mathscr{D} f, a\rangle=0
$$

is a consequence of these conditions [Roytenberg 1999].

A smooth subbundle $L$ of a Courant algebroid is called a Dirac subbundle if it is a maximal isotropic, with respect to $\langle\cdot, \cdot\rangle$, and its space of sections $\Gamma(L)$ is closed under $\llbracket \cdot, \cdot \rrbracket$. While not all Courant algebroids are Lie algebroids (since the Jacobi identity is not satisfied), their Dirac subbundles are Lie algebroids.

Example 3.1 ([Courant 1990]). Given a smooth manifold $M$, the bundle $T M \rightarrow M$ carries a natural Courant algebroid structure, where the anchor is the identity map and the pairing and bracket are given, respectively, by

$$
\begin{aligned}
& \langle X+\xi, Y+\eta\rangle=\frac{1}{2}(\xi(Y)+\eta(X)), \\
& \llbracket X+\xi, Y+\eta \rrbracket=[X, Y]+\mathscr{L}_{X} \eta-\mathscr{L}_{Y} \xi+\frac{1}{2} d(\xi(Y)-\eta(X)),
\end{aligned}
$$

for all $X, Y \in \mathfrak{X}^{\bullet}(M)$, and for all $\xi, \eta \in \Omega^{1}(M)$.

Let $E$ be a Courant algebroid on a smooth manifold $M$. And let

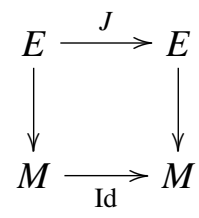


be a vector bundle map such that $J^{2}=-$ Id. Then the complexification $E_{\mathbb{C}}:=$ $E \otimes \mathbb{C}$ - with the extended $\mathbb{C}$-linear Courant algebroid structure - decomposes as the direct sum $L \oplus \bar{L}$ of the eigenbundles of $J$. Here $L$ is associated to the eigenvalue $+i$ and its complex conjugate $\bar{L}$ to $-i$. The bundle map $J$ is called a generalized complex structure if $J$ is orthogonal with respect to $\langle\cdot, \cdot\rangle$ - this forces $L$ and $\bar{L}$ to be isotropic - and the spaces of sections $\Gamma(L)$ and $\Gamma(\bar{L})$ are closed under the Courant bracket, or equivalently, $J$ is "integrable":

$$
\llbracket J x, J y \rrbracket-\llbracket x, y \rrbracket-J(\llbracket J x, y \rrbracket+\llbracket x, J y \rrbracket)=0, \quad \text { for all } x, y \in \Gamma(E) .
$$

Since the pairing is nondegenerate, the map

$$
\Xi: E \stackrel{\text { iso }}{\longrightarrow} E^{*}, e \mapsto\langle e, \cdot\rangle
$$

is an isomorphism of vector bundles. One has $\Xi^{*}=\Xi$ (modulo the canonical isomorphism $\left(E^{*}\right)^{*}=E$ ) and $\Xi \circ J+J^{*} \circ \Xi=0$.

Proposition 3.2. The bracket

$$
\{f, g\}=2\langle J \mathscr{D} f, \mathscr{D} g\rangle, \quad f, g \in C^{\infty}(M)
$$

is a Poisson structure on the manifold $M$.

Gualtieri [2007] proved a formula similar to (but slightly less general than) (3-8).

Proof. It is easy to see that this bracket is a skew-symmetric derivation of $C^{\infty}(M)$. It remains to check that the Jacobi identity is satisfied. Since $J$ is integrable, we have

$$
\llbracket J \mathscr{D} f, J \mathscr{D} g \rrbracket-\llbracket \mathscr{D} f, \mathscr{D} g \rrbracket-J(\llbracket J \mathscr{D} f, \mathscr{D} g \rrbracket+\llbracket \mathscr{D} f, J \mathscr{D} g \rrbracket)=0,
$$

for all $f, g \in C^{\infty}(M)$. Pairing with $\mathscr{D} h$, we obtain

$$
\langle\llbracket J \mathscr{D} f, J \mathscr{D} g \rrbracket, \mathscr{D} h\rangle-\langle\llbracket \mathscr{D} f, \mathscr{D} g \rrbracket, \mathscr{D} h\rangle-\langle J(\llbracket J \mathscr{D} f, \mathscr{D} g \rrbracket+\llbracket \mathscr{D} f, J \mathscr{D} g \rrbracket), \mathscr{D} h\rangle=0 .
$$

We compute the first term of (3-9):

$$
\begin{array}{ll}
\langle\llbracket J \mathscr{D} f, J \mathscr{D} g \rrbracket, \mathscr{D} h\rangle & \\
=\langle\llbracket J \mathscr{D} f, J \mathscr{D} g \rrbracket+\mathscr{D}\langle J \mathscr{D} f, J \mathscr{D} g\rangle, \mathscr{D} h\rangle & \text { by (3-5) } \\
=\rho(J \mathscr{D} f)\langle J \mathscr{D} g, \mathscr{D} h\rangle-\langle J \mathscr{D} g, \llbracket J \mathscr{D} f, \mathscr{D} h \rrbracket+\mathscr{D}\langle J \mathscr{D} f, \mathscr{D} h\rangle\rangle & \text { by (3-6) } \\
=2\langle\mathscr{J}(J g, \mathscr{D} h\rangle, J \mathscr{D} f\rangle-\langle J \mathscr{D} g, \mathscr{D}\langle\mathscr{D} h, J \mathscr{D} f\rangle+\mathscr{D}\langle J \mathscr{D} f, \mathscr{D} h\rangle\rangle & \text { by (3-1), (3-7) } \\
=2\{f,\{g, h\}\}-2\{g,\{f, h\}\} & \text { by (3-8) } \\
=2\{f,\{g, h\}\}+2\{g,\{h, f\}\} . &
\end{array}
$$

By (3-1), (3-2) and (3-5), the second term of (3-9) vanishes. Finally, the third term of (3-9) gives 


$$
\begin{aligned}
-\langle J(\llbracket J \mathscr{D} f, \mathscr{D} g \rrbracket & +\llbracket \mathscr{D} f, J \mathscr{D} g \rrbracket), \mathscr{D} h\rangle \\
& =-\langle J(\mathscr{D}\langle\mathscr{D} g, J \mathscr{D} f\rangle-\mathscr{D}\langle\mathscr{D} f, J \mathscr{D} g\rangle), \mathscr{D} h\rangle \\
& =-\{\{f, g\}, h\}+\{\{g, f\}, h\} \\
& =2\{h,\{f, g\}\}
\end{aligned}
$$

Proposition 3.3. Let $\Pi$ denote the bivector field on $M$ associated to the Poisson bracket (3-8). We have

$$
\Pi^{\sharp}=\frac{1}{2} \rho \circ J \circ \Xi^{-1} \circ \rho^{*},
$$

where $\Pi^{\sharp}: T^{*} M \rightarrow T M$ is the vector bundle map equivalent to $\Pi \in \Gamma\left(\wedge^{2} T^{*} M\right)$ via $\Pi(\alpha, \beta)=\beta\left(\Pi^{\sharp} \alpha\right)$, for all $\alpha, \beta \in \Omega^{1}(M)$. The Hamiltonian vector field associated to the function $f \in C^{\infty}(M)$ is

$$
X_{f}=\Pi^{\sharp} d f=\rho \circ J \circ \mathscr{D} f .
$$

And the characteristic distribution is

$$
\Pi^{\sharp}\left(T^{*} M\right)=\rho J(\operatorname{ker} \rho)^{\perp},
$$

where $(\operatorname{ker} \rho)^{\perp}$ refers to the subbundle of $E$ orthogonal to $\operatorname{ker} \rho$ with respect to $\langle\cdot, \cdot\rangle$.

Proof. One has

$$
X_{f}(g)=\{f, g\}=2\langle J \mathscr{D} f, \mathscr{D} g\rangle=\rho(J \mathscr{D} f)(g)
$$

and

$$
\Pi^{\sharp}(d f)=X_{f}=\rho J ゆ f=\frac{1}{2} \rho J \Xi^{-1} \rho^{*}(d f)
$$

since (3-1) can be reinterpreted as $\langle\mathscr{D} f, \cdot\rangle=\frac{1}{2} \Xi^{-1} \rho^{*} d f$.

Proposition 3.4. If $E=T M$ is the standard Courant algebroid of Example 3.1 and the matrix representation of $J$ relative to the above direct sum decomposition is shown in (1-3), then $\Pi=\pi$.

Proof. Here $\mathscr{D}$ coincides with the de Rham differential $d$. Thus

$$
\{f, g\}_{\Pi}=2\langle J \mathscr{D} f, \mathscr{D} g\rangle=2\left\langle\pi^{\sharp} d f-\phi^{*} d f, d g\right\rangle=d g\left(\pi^{\sharp} d f\right)=\{f, g\}_{\pi} .
$$

Recall that the complexification of $E$ decomposes as the direct sum $E_{\mathbb{C}}=L_{+} \oplus$ $L_{-}$, where $L_{ \pm}$are Dirac structures (with anchor maps $\rho_{ \pm}$). Thus $\left(L_{+}, L_{-}\right)$is a complex Lie bialgebroid [Mackenzie and Xu 1994], where $L_{ \pm}^{*}$ is identified with $L_{\mp}$ via $\Xi$. As shown in [Mackenzie and Xu 1994, Proposition 3.6], to any complex Lie bialgebroid is associated a complex bivector field $\varpi$ on $M$ given by

$$
i \varpi^{\sharp}=\rho_{-} \circ \Xi^{-1} \circ \rho_{+}^{*}=-\rho_{+} \circ \Xi^{-1} \circ \rho_{-}^{*} .
$$


Lemma 3.5. The Poisson bivector $\varpi$ coming from the Lie bialgebroid structure $\left(L_{+}, L_{-}\right)$is real and coincides with $\Pi$.

Proof. It suffices to observe that the following two compositions are both equal to $\varpi^{\sharp}:$

$$
\begin{aligned}
& T_{\mathbb{C}} M \stackrel{\rho_{-}}{\longleftarrow} L_{-} \stackrel{-i \cdot}{\longleftarrow} L_{-} \stackrel{\Xi^{-1}}{\longleftarrow} L_{+}^{*} \stackrel{\rho_{+}^{*}}{\longleftarrow} T_{\mathbb{C}}^{*} M \\
& T_{\mathbb{C}} M \stackrel{\rho_{+}}{\longleftarrow} L_{+} \stackrel{+i \cdot}{\longleftarrow} L_{+} \stackrel{\Xi^{-1}}{\longleftarrow} L_{-}^{*} \stackrel{\rho_{-}^{*}}{\longleftarrow} T_{\mathbb{C}}^{*} M
\end{aligned}
$$

and that their sum

$$
T_{\mathbb{C}} M \stackrel{\rho}{\leftarrow} E_{\mathbb{C}} \stackrel{J}{\longleftarrow} E_{\mathbb{C}} \stackrel{\Xi^{-1}}{\longleftarrow} E_{\mathbb{C}}^{*} \stackrel{\rho^{*}}{\longleftarrow} T_{\mathbb{C}}^{*} M
$$

is equal to $2 \Pi^{\sharp}$.

Proposition 3.6. $\rho\left(L_{+}\right) \cap \rho\left(L_{-}\right)=\Delta \otimes \mathbb{C}$ with $\Delta=\rho(J \operatorname{ker} \rho)$

Proof. If $v \in \rho\left(L_{+}\right) \cap \rho\left(L_{-}\right)$, then $\bar{v} \in \rho\left(L_{-}\right) \cap \rho\left(L_{+}\right)$. Hence there exists a subbundle $\Delta$ of $T M$ such that $\rho\left(L_{+}\right) \cap \rho\left(L_{-}\right)=\Delta \otimes \mathbb{C}$. For all $k \in \operatorname{ker} \rho$, one has $\rho\left(L_{+}\right) \ni \rho\left(\frac{1+i J}{2} k\right)=\frac{i}{2} \rho(J k)$. Therefore, $\rho(J \operatorname{ker} \rho) \subset \rho\left(L_{+}\right)$. Since $J$ is real, $\rho(J \operatorname{ker} \rho) \subset \rho\left(L_{+}\right) \cap \rho(E)=\Delta$. It remains to prove the converse inclusion: $\Delta \subset \rho(J \operatorname{ker} \rho)$. Since $\Delta=\rho\left(L_{+}\right) \cap \rho(E)$, given $\delta \in \Delta$, there exists $l_{+} \in L_{+}$such that $\rho\left(l_{+}\right)=\delta=\rho\left(\overline{l_{+}}\right)$. Thus $\delta=\rho\left(\frac{l_{+} \overline{l_{+}}}{2}\right)=\rho\left(J\left(\frac{l_{+}-\overline{l_{+}}}{2 i}\right)\right)$ with $\frac{l_{+}-\overline{l_{+}}}{2 i} \in \operatorname{ker} \rho$.

Remark 3.7. If $E=T M$ is the standard Courant algebroid of Example 3.1, then $(\operatorname{ker} \rho)^{\perp}=T^{*} M=\operatorname{ker} \rho$. Therefore, in this particular case, $\Pi^{\sharp}\left(T_{\mathbb{C}}^{*} M\right)=\rho\left(L_{+}\right) \cap$ $\rho\left(L_{-}\right)$, recovering Gualtieri's result Gualtieri [2003].

It would be interesting to explore when the symplectic foliation $\Pi^{\sharp}\left(T_{\mathbb{C}}^{*} M\right)$ coincides with $\rho\left(L_{+}\right) \cap \rho\left(L_{-}\right)$for arbitrary Courant algebroids.

\section{The induced generalized complex structure}

Consider two twisted manifolds $(M, \Omega)$ and $(N, \Upsilon)$ with an immersion $h: N \rightarrow M$. Also, assume that there is a generalized complex structure $J$ on $M$ with eigenbundles $L_{+}$and $L_{-}$. The goal of this section is to characterize when the pull backs of $L_{+}$and $L_{-}$give a generalized complex structure on $N$. The pull backs of $L_{+}$and $L_{-}$will be called the induced bundles, and are given by

$$
L_{ \pm}^{\prime}=\mathscr{B}_{h}\left(L_{ \pm}\right)=\left\{X+h^{*} \xi: X \in T_{\mathbb{C}} N, \xi \in T_{\mathbb{C}}^{*} M \text { such that } h_{*} X+\xi \in L_{ \pm}\right\} .
$$

By definition, both $L_{+}^{\prime}$ and $L_{-}^{\prime}$ are maximal isotropics, but they need not be smooth or involutive subbundles. The bundles may also have nontrivial intersection. The rest of this section is devoted to characterizing when the induced bundles have the desired properties. The first of these properties to be addressed will be the intersection property. 
Because $L_{+}^{\prime}$ and $L_{-}^{\prime}$ are both maximal isotropics, it suffices to check that they span $T_{\mathbb{C}} N \oplus T_{\mathbb{C}}^{*} N$. Consider the subbundle $B=\left.T N \oplus T^{*} M\right|_{N}$ of $T M \oplus T^{*} M$. Its orthogonal, $B^{\perp}=T N^{o}$, is the kernel of the natural projection $s: B \rightarrow T N \oplus T^{*} N$, which maps $X+\xi \mapsto X+h^{*} \xi$. It is not hard to see that $s((B \cap J B) \otimes \mathbb{C})=$ $L_{+}^{\prime}+L_{-}^{\prime}$. Thus the decomposition, $T_{\mathbb{C}} N \oplus T_{\mathbb{C}}^{*} N=L_{+}^{\prime} \oplus L_{-}^{\prime}$, holds if and only if $B=B \cap J B+B^{\perp}$. The preceding can be summarized in the following proposition.

Proposition 4.1. The following assertions are equivalent.

(1) The subbundle $L_{+}^{\prime}$ is the $+i$-eigenbundle of a-not necessarily smoothautomorphism $J^{\prime}$ of $T N \oplus T^{*} N$ such that $J^{\prime 2}=-\operatorname{Id}$ and $J^{\prime} J^{\prime *}=$ Id.

(2) $B=B \cap J B+B^{\perp}$.

(3) $J B \subseteq B+J B^{\perp}$.

(4) $J B^{\perp} \cap B \subseteq B^{\perp}$.

Conditions (3) and (4) follow from elementary calculations. In the sequel we will assume that the assertions of Proposition 4.1 are satisfied. Consider the restriction of $J$ and $s$ to the $J$-invariant subspace $B \cap J B$; the latter map will be denoted by $s^{\prime}$. The kernel of $s^{\prime}$ is $B^{\perp} \cap J B$. Under $J$, this kernel is mapped to $J B^{\perp} \cap B$. This must be in $J B \cap B$ and also, by Proposition 4.1, in $B^{\perp}$, however $B^{\perp} \subseteq B$. So the image of the kernel of $s^{\prime}$ is in $B^{\perp} \cap J B \cap B=B^{\perp} \cap J B$. Thus the kernel of $s^{\prime}$ is $J$-invariant and $\left.J\right|_{B \cap J B}$ induces an automorphism of $T N \oplus T^{*} N$ :

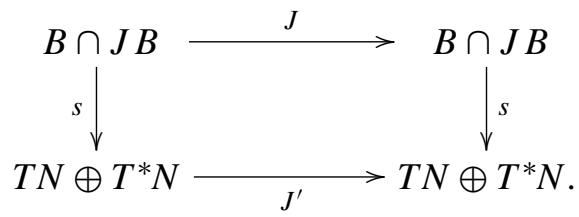

The induced automorphism is nothing but $J^{\prime}$ from Proposition 4.1. Indeed, the complexification of the above commutative diagram gives

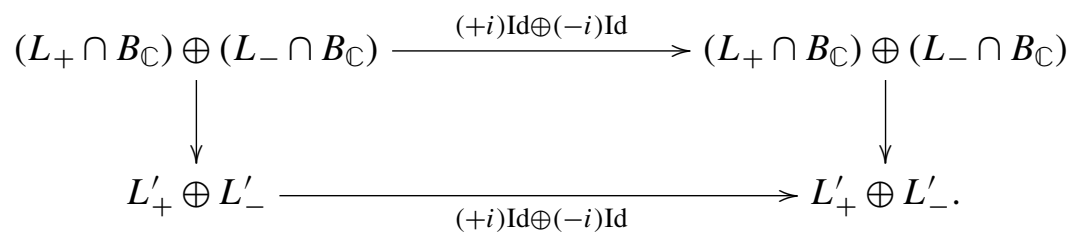

The next lemma relates condition (4) of Proposition 4.1 to the splitting of $J$ :

$$
\left(\begin{array}{cc}
\phi & \pi^{\sharp} \\
\sigma_{b} & -\phi^{*}
\end{array}\right)
$$

Lemma 4.2. The following assertions are equivalent.

(1) $J B^{\perp} \cap B \subseteq B^{\perp}$.

(2) $T N \cap \pi^{\sharp}\left(T N^{o}\right)=0$ and $\phi(T N) \subseteq T N+\pi^{\sharp}\left(T N^{o}\right)$. 
Proof. The inclusion $J\left(T N^{o}\right) \cap\left(\left.T N \oplus T^{*} M\right|_{N}\right) \subseteq T N^{o}$ is true if, and only if,

$$
\left\{\begin{array}{l}
\xi \in T N^{o} \\
\left.J \xi \in T N \oplus T^{*} M\right|_{N}
\end{array}\right\} \Longrightarrow J \xi \in T N^{o}
$$

if, and only if,

$$
\left\{\begin{array}{l}
\xi \in T N^{o} \\
\pi^{\sharp} \xi \in T N
\end{array}\right\} \Longrightarrow\left\{\begin{array}{l}
\pi^{\sharp} \xi=0 \\
\phi^{*} \xi \in T N^{o}
\end{array}\right\}
$$

if, and only if,

$$
\xi \in T N^{o} \cap\left(\pi^{\sharp}\right)^{-1}(T N) \Longrightarrow\left\{\begin{array}{l}
\pi^{\sharp} \xi=0 \\
\xi \in(\phi(T N))^{o}
\end{array}\right\}
$$

if, and only if,

$$
\pi^{\sharp}\left(T N^{o}\right) \cap T N=0 \quad \text { and } \quad T N^{o} \cap\left(\pi^{\sharp}\right)^{-1}(T N) \subseteq(\phi(T N))^{o} .
$$

Since $\pi$ is skew-symmetric $\left(\pi^{\sharp}\right)^{-1}(T N)=\left(\pi^{\sharp}\left(T N^{o}\right)\right)^{o}$, and

$$
\begin{gathered}
T N^{o} \cap\left(\pi^{\sharp}\left(T N^{o}\right)\right)^{o} \subseteq(\phi(T N))^{o} \\
T N+\pi^{\sharp}\left(T N^{o}\right) \supseteq \phi(T N) .
\end{gathered}
$$

According to this lemma, the sum $T N+\pi^{\sharp}\left(T N^{o}\right)$ must be direct. In the sequel pr will denote the projection $T N \oplus \pi^{\sharp}\left(T N^{o}\right) \rightarrow T N$. If $\pi$ is degenerate then neither the bundle $T N \oplus \pi^{\sharp}\left(T N^{o}\right)$, nor the map pr is necessarily smooth.

For any $\xi \in T^{*} N$ we claim that if $\eta, \eta^{\prime} \in B \cap J B$ such that $\xi=h^{*} \eta=h^{*} \eta^{\prime}$ then $\pi^{\sharp} \eta=\pi^{\sharp} \eta^{\prime}$. Because $\eta$ and $\eta^{\prime}$ are preimages of $\xi$ they differ by some element of $T N^{o}$, and as $B \cap J B$ is $J$-stable both $\pi^{\sharp} \eta$ and $\pi^{\sharp} \eta^{\prime}$ are in $T N$. However $T N \cap \pi^{\sharp}\left(T N^{o}\right)=\{0\}$, and the difference of the two preimages is zero. Thus the assignment $\xi \mapsto \pi^{\sharp} \eta$ defines a skew-symmetric vector bundle map from $T^{*} N$ to $T N$. Its associated bivector field on $N$ will be denoted by $\pi^{\prime}$.

The following technical lemmas will be used to show when $J^{\prime}$ is smooth.

Lemma 4.3. Let $X \in T N$ and $\xi \in T N^{o}$. If $\phi X+\pi^{\sharp} \xi \in T N$ then $\phi X+\pi^{\sharp} \xi=$ $(\operatorname{pr} \circ \phi) X$.

Proof. For any $X \in T N$ the second assertion of Lemma 4.2 gives $\phi X=Y+\pi^{\sharp} \eta$, where $Y \in T N$ and $\eta \in T N^{o}$. By definition $Y=(\operatorname{pr} \circ \phi) X$, and $\phi X+\pi^{\sharp} \xi=$ $Y+\pi^{\sharp}(\eta+\xi)$. Both $Y$ and $\phi X+\pi^{\sharp} \xi$ are elements of $T N$; thus $\pi^{\sharp}(\eta+\xi)$ is also an element of $T N$. But $T N \cap \pi^{\sharp}\left(T N^{o}\right)=\{0\}$, and $\eta+\xi \in T N^{o}$. Thus $\pi^{\sharp}(\eta+\xi)=0$.

Lemma 4.4. Let $p_{1}: T N \oplus T^{*} N \rightarrow T N$ and $p_{2}: T N \oplus T^{*} N \rightarrow T^{*} N$ be the projections. If $X \in T N$, then

$$
\left(p_{1} J^{\prime}\right) X=(\operatorname{pr} \circ \phi) X=\phi X+\pi^{\sharp} \zeta
$$


and

$$
\left(p_{2} J^{\prime}\right) X=h^{*}\left(\sigma_{b} X-\phi^{*} \zeta\right),
$$

where $\zeta$ is some element of $T N^{o}$ such that $X+\zeta \in B \cap J B$.

If $\xi \in T^{*} N$, then

$$
\left(p_{1} J^{\prime}\right) \xi=\pi^{\prime \sharp} \xi=\pi^{\sharp} \eta
$$

and

$$
\left(p_{2} J^{\prime}\right) \xi=-\left(h^{*} \phi^{*}\right) \eta,
$$

where $\eta$ is some element of $\left.T^{*} M\right|_{N} \cap B \cap J B$ such that $h^{*} \eta=\xi$.

Proof. Consider $X \in T N$. Since $s$ is surjective there exists some $\zeta \in T N^{o}$ such that $X+\zeta \in B \cap J B$ and $s(X+\zeta)=X$. Now $J(X+\zeta)=\left(\phi X+\pi^{\sharp} \zeta\right)+\left(\sigma_{b} X-\phi^{*} \zeta\right) \in B$. Therefore $\phi X+\pi^{\sharp} \zeta \in T N$ and, by Lemma 4.3, $\phi X+\pi^{\sharp} \zeta=($ pro $\phi) X$. Both (4-2) and (4-3) follow from (4-1).

Now take $\xi \in T^{*} N$. Again, since $s$ is surjective there exists some $\eta \in T^{*} M$ such that $\eta \in B \cap J B$ and $s(\eta)=\xi$. Now $J(\xi)=\pi^{\sharp} \eta-\phi^{*} \eta=\pi^{\prime \prime} \xi-\phi^{*} \eta$, which is in $B$. Both (4-4) and (4-5) follow from (4-1).

For the remainder of this section, if $L$ is a smooth vector bundle then $\Gamma(L)$ will denote the space of all — not necessarily smooth — sections of $L$, and $\Gamma^{\infty}(L)$ the subspace of smooth sections.

Lemma 4.5. Let $\xi \in \Gamma\left(T N^{o}\right)$. If $\pi^{\sharp} \xi \in \Gamma^{\infty}\left(\left.T M\right|_{N}\right)$, then $\left(h^{*} \phi^{*}\right) \xi \in \Gamma^{\infty}\left(T^{*} N\right)$.

Proof. As noted previously, if $X \in \Gamma^{\infty}(T N)$ then $\phi X=Y+\pi^{\sharp} \eta$, where $Y \in \Gamma(T N)$ and $\eta \in \Gamma^{\infty}\left(T N^{o}\right)$. Now $\left(\phi^{*} \xi\right)(X)=\xi(\phi X)=\xi(Y)+\xi\left(\pi^{\sharp} \eta\right)=\xi\left(\pi^{\sharp} \eta\right)=-\eta\left(\pi^{\sharp} \xi\right)$. This function and its restriction to $T N$ are smooth because $\pi^{\sharp} \xi$ is.

Lemma 4.6. Assume $\mathrm{pr} \circ \phi$ is a smooth map and $\eta \in \Gamma\left(\left.T^{*} M\right|_{N}\right)$. If $h^{*} \eta \in \Gamma^{\infty}(T N)$ and $\pi^{\sharp} \eta \in \Gamma^{\infty}(T N)$ then $\left(h^{*} \phi^{*}\right) \eta \in \Gamma^{\infty}\left(T^{*} N\right)$.

Proof. Once again, if $Y \in \Gamma^{\infty}(T N)$ then

$$
\phi Y=(\operatorname{pr} \circ \phi) Y+\pi^{\sharp} \zeta
$$

for some $\zeta \in \Gamma^{\infty}\left(T N^{o}\right)$. Now

$$
\begin{aligned}
& \left(\left(h^{*} \phi^{*}\right) \eta\right)(Y)=\left(h^{*} \eta\right)(\phi Y)=\left(h^{*} \eta\right)((\operatorname{pr} \circ \phi) Y)+\left(h^{*} \eta\right)\left(\pi^{\sharp} \zeta\right) \\
= & \left(h^{*} \eta\right)((\operatorname{pr} \circ \phi) Y)-\zeta\left(\pi^{\sharp}\left(h^{*} \eta\right)\right) .
\end{aligned}
$$

Thus $\left(\left(h^{*} \phi^{*}\right) \eta\right)(Y)$ is a smooth function, and the lemma follows.

We are now ready to give the conditions $J^{\prime}$ must satisfy in order to be smooth. 
Proposition 4.7. The vector bundle automorphism $J^{\prime}$ of $T N \oplus T^{*} N$ is smooth if, and only if, pro $\phi: T N \rightarrow T N$ is smooth and $\pi^{\prime}$ is a smooth bivector field on $N$.

Proof. First assume that $J^{\prime}$ is smooth. Thus $\left(p_{1} J^{\prime}\right) X \in \Gamma^{\infty}(T N)$ for all $X \in$ $\Gamma^{\infty}(T N)$. It follows from (4-2) that (pro $\left.\phi\right)$ must be smooth. Also $\left(p_{1} J^{\prime}\right) \xi \in$ $\Gamma^{\infty}\left(T^{*} N\right)$ for all $\xi \in \Gamma^{\infty}\left(T^{*} N\right)$, and (4-4) shows that $\pi^{\prime \sharp}$ is smooth.

Now for the other implication. For every $X \in \Gamma^{\infty}\left(T^{*} N\right)$ there is some $\zeta \in$ $\Gamma\left(T N^{o}\right)$ such that (4-2) and (4-3) are satisfied. As $J$ is smooth both $\sigma_{b}$ and $\phi$ are smooth. The smoothness of $\operatorname{pr} \circ \phi$ and (4-2) show that $\pi^{\sharp} \zeta \in \Gamma^{\infty}\left(\left.T M\right|_{N}\right)$. Thus, according to Lemma 4.5, $\left(h^{*} \phi^{*}\right) \zeta \in \Gamma^{\infty}\left(T^{*} N\right)$, and the right hand sides of (4-2) and (4-3) are smooth. Finally $J^{\prime} X=\left(p_{1} J^{\prime}\right) X+\left(p_{2} J^{\prime}\right) X \in \Gamma^{\infty}\left(T N \oplus T^{*} N\right)$.

Now take $\xi \in \Gamma^{\infty}\left(T^{*} N\right)$. There must exist $\eta \in \Gamma\left(\left.T^{*} M\right|_{N}\right)$ such that (4-4) holds, (4-5) holds, and $h^{*} \eta=\xi$. The smoothness of $\pi^{\prime}$ and (4-4) show that $\pi^{\sharp} \eta \in \Gamma^{\infty}(T N)$. Now Lemma 4.6 gives $\left(h^{*} \phi^{*}\right) \eta \in \Gamma^{\infty}\left(T^{*} N\right)$, and the right hand sides of (4-4) and (4-5) are smooth. Finally,

$$
J^{\prime} \xi=\left(p_{1} J^{\prime}\right) \xi+\left(p_{2} J^{\prime}\right) \xi \in \Gamma^{\infty}\left(T N \oplus T^{*} N\right) .
$$

We finish this section by using Lemma 2.1 to show when $J^{\prime}$ is integrable.

Proposition 4.8. If $J^{\prime}$ is smooth then it is integrable.

Proof. First, observe that the vector bundles $L_{ \pm} \cap B_{\mathbb{C}}=(I \mp i J) B_{\mathbb{C}}$ are smooth. Since $J^{\prime}$ is smooth, its eigenbundles $L_{ \pm}^{\prime}$ are also smooth. It is not hard to check that any smooth section of $L_{+}^{\prime}$ is $h$-related to a smooth section of $L_{+} \cap B_{\mathbb{C}}$.

Hence for any $\sigma_{1}^{\prime}, \sigma_{2}^{\prime} \in \Gamma^{\infty}\left(L_{+}^{\prime}\right)$ there exists $\sigma_{1}, \sigma_{2} \in \Gamma^{\infty}\left(L_{+} \cap B_{\mathbb{C}}\right)$ such that $\sigma_{1} \stackrel{h}{h} \sigma_{1}^{\prime}$ and $\sigma_{2} \rightsquigarrow \sigma_{2}^{\prime}$. Since $L_{+}$is integrable $\llbracket \sigma_{1}, \sigma_{2} \rrbracket_{\Omega} \in \Gamma^{\infty}\left(L_{+}\right)$, and it follows from Lemma 2.1 that $\llbracket \sigma_{1}, \sigma_{2} \rrbracket_{\Omega} \stackrel{h}{\rightsquigarrow} \llbracket \sigma_{1}^{\prime}, \sigma_{2}^{\prime} \rrbracket_{\Upsilon}$. Thus $\llbracket \sigma_{1}^{\prime}, \sigma_{2}^{\prime} \rrbracket_{\Upsilon} \in \Gamma^{\infty}\left(L_{+}^{\prime}\right)$, and $L_{+}^{\prime}$ is involutive with respect to the $\Upsilon$-twisted bracket.

\section{Main theorem}

The following definition will be used to characterize when a twisted submanifold is also generalized complex; see [Crainic and Fernandes 2004] for the motivation of this definition.

Definition 5.1. Let $(M, \pi)$ be a Poisson manifold. A smooth submanifold $N$ of $M$ is a Poisson-Dirac submanifold of $M$ if $T N \cap \pi^{\sharp}(T N)^{o}=\{0\}$, and the induced Poisson tensor $\pi^{\prime}$ on $N$ is smooth.

The next theorem is the main result of this paper. The untwisted version of this result was obtained independently, using a different method, by Vaisman [2007].

Theorem 5.2. Let $(M, \Omega, J)$ be a twisted generalized complex manifold with $J=$ $\left(\begin{array}{cc}\phi & \pi^{\sharp} \\ \sigma_{b} & -\phi^{*}\end{array}\right)$. A twisted submanifold $N$ of $M$ inherits a twisted generalized complex 
structure $J^{\prime}$, making it a twisted generalized complex submanifold, if and only the following conditions hold:

(1) $N$ is a Poisson-Dirac submanifold of $(M, \pi)$.

(2) $\phi(T N) \subseteq T N+\pi^{\sharp}\left(T N^{o}\right)$.

(3) $\operatorname{pr} \circ \phi: T N \rightarrow T N$ is smooth.

The generalized complex structure $J^{\prime}$ on $N$ is given by

$$
J^{\prime}=\left(\begin{array}{cc}
\phi^{\prime} & \left(\pi^{\prime}\right)^{\sharp} \\
\sigma_{b}^{\prime} & -\left(\phi^{\prime}\right)^{*}
\end{array}\right) \text {. }
$$

Here $\phi^{\prime}=\left.\operatorname{pr} \circ \phi\right|_{T N}, \pi^{\prime}$ is the induced Poisson tensor, and

$$
\sigma_{b}^{\prime}(X)=h^{*}\left(\sigma_{b} X-\phi^{*} \zeta\right)
$$

where $\zeta \in(T N)^{o}$ such that $X+\zeta \in B \cap J B$, as in Lemma 4.4 .

Proof. This theorem is the construction and confirmation of the properties of $J^{\prime}$. Proposition 4.1 combined with Lemma 4.2 shows that $J^{\prime 2}=-\mathrm{Id}$ and $J^{\prime *} J^{\prime}=\mathrm{Id}$. The smoothness of $J^{\prime}$ follows from Proposition 4.7, and the integrability of its $+i$ eigenbundle follows from Proposition 4.8. The form of the generalized complex structure follows from Lemma 4.4.

For the following examples let $\Omega=0$.

Example 5.3. Let $(M, j)$ be a complex manifold, and let $N$ be a smooth submanifold of $M$. There is a generalized complex structure on $M$ given by $\phi=j, \sigma=0$ and $\pi=0$. Because the Poisson structure is zero, $N$ is automatically a PoissonDirac submanifold. Condition (2) of Theorem 5.2 becomes $j(T N) \subseteq T N$, which is exactly the requirement for $N$ to be an immersed complex submanifold of $M$. Now $\operatorname{pr} \circ j=\left.j\right|_{T N}$, which is a smooth map. Thus $N$ is a generalized complex submanifold if, and only if, it is an immersed complex submanifold.

Example 5.4. Let $(M, \omega)$ be a symplectic manifold and $N$ a smooth submanifold of $M$. The generalized complex structure on $M$ arising from $\omega$ is given by $\phi=0$, $\sigma_{b}=\omega_{b}$ and $\pi^{\sharp}=-\omega_{b}{ }^{-1}$. Because $\phi=0$, conditions (2) and (3) of Theorem 5.2 are automatically satisfied. Now $N$ will be a Poisson-Dirac submanifold of $M$ if, and only if, $N$ is a symplectic submanifold of $M$. Thus $N$ is a generalized complex submanifold of $M$ if, and only if, it is a symplectic submanifold.

The last result of this section is an application of Theorem 5.2 to the stable locus of a twisted generalized complex involution. This result is similar to one for Poisson involutions [Fernandes and Vanhaecke 2001; Xu 2003]. Let $(M, \Omega, J)$ be 
a twisted generalized complex manifold. A twisted generalized complex involution is a diffeomorphism $\Psi: M \rightarrow M$ such that $\Psi^{2}=\mathrm{Id}$ and

$$
\Psi_{*}^{*} \circ J=J \circ \Psi_{*}^{*} .
$$

Here $\Psi_{*}^{*}$ is the map from $T M \oplus T^{*} M$ to $T M \oplus T^{*} M$ defined by $\Psi_{*}^{*}(X+\xi)=$ $\Psi_{*} X+\Psi^{*} \xi$.

Corollary 5.5. Let $(M, \Omega, J)$ be a twisted generalized complex manifold and let $\Psi$ be a twisted generalized complex involution of $J$. The fixed locus, $N$, of $\Psi$ is a twisted generalized complex submanifold of $M$.

Proof. Let $\xi$ be an arbitrary element of $T^{*} M$. (5-1) implies that $\left(\Psi_{*} \pi^{\sharp} \Psi^{*}\right) \xi=$ $\pi \sharp \xi$. Hence $\Psi_{*} \pi=\pi$, and $\Psi_{*}$ is a Poisson involution. Because $\Psi_{*}$ is a Poisson involution, [Xu 2003, Proposition 4.1] implies that $N$ is a Dirac submanifold. Thus $N$ is a Poisson-Dirac submanifold, and condition (1) of Theorem 5.2 is satisfied.

Take $X \in T N$. Equation (5-1) implies that $\Psi_{*}(\phi X)+\Psi^{*}\left(\sigma_{b} X\right)=\phi X+\sigma_{b} X$. The vector field component of this equality proves that $\phi(T N) \subseteq T N$, and condition (2) of Theorem 5.2 is satisfied. Thus pr $\circ \phi=\left.\phi\right|_{T N}$, which is a smooth map. Hence condition (3) of Theorem 5.2 is satisfied.

\section{Holomorphic Poisson submanifolds}

Let $(M, j, \pi)$ be a Poisson Nijenhuis manifold such that $j: T M \rightarrow T M$ is an integrable almost complex structure. Such a structure is equivalent to a holomorphic Poisson structure The holomorphic Poisson tensor is given by $\Pi=\pi_{j}+i \pi$, where $\pi_{j}^{\sharp}=\pi^{\sharp} \circ j^{*}$.

A generalized complex structure on $M$ [Crainic 2004; Stiénon and Xu 2007] is given by

$$
J=\left(\begin{array}{cc}
j & \pi^{\sharp} \\
0 & -j^{*}
\end{array}\right) .
$$

In general, if $N$ is a generalized complex submanifold then the induced generalized complex need not have $\sigma^{\prime}=0$.

Recall that $T N \cap \pi^{\sharp}\left(T N^{o}\right)=\{0\}$ and $\phi(T N) \subseteq T N+\pi^{\sharp}\left(T N^{o}\right)$. Thus, we can define the composition

$$
T N \stackrel{\phi}{\longrightarrow} T N \oplus \pi^{\sharp}\left(T N^{o}\right) \stackrel{\mathrm{pr}_{2}}{\longrightarrow} \pi^{\sharp}\left(T N^{o}\right) .
$$

Proposition 6.1. Consider the generalized complex structure (6-1), and let $N$ be a generalized complex submanifold of $M$. Now, $\sigma^{\prime}=0$ if, and only if,

$$
\phi(T N) \subseteq \pi^{\sharp}\left(\left(\mathrm{pr}_{2} \circ \phi(T N)\right)^{o}\right) .
$$


Proof. Take $X \in T N$. Then $\left.\left(\begin{array}{c}X \\ 0\end{array}\right) \in T N \oplus T^{*} M\right|_{N}=B$. Since $B=B \cap J B+B^{\perp}$ and $B^{\perp}=T N^{o}$ there exists a $\zeta \in T N^{o}$ such that $\left(\begin{array}{c}X \\ \zeta\end{array}\right) \in B \cap J B$. But then $J\left(\begin{array}{c}X \\ \zeta\end{array}\right) \in B$ too, and so $\phi X+\pi^{\sharp} \zeta \in T N$.

In other words, given $X \in T N$ there exists $\zeta \in T N^{o}$ such that $\phi X+\pi^{\sharp} \zeta \in T N$. Since $\phi(T N) \subseteq T N \oplus \pi^{\sharp}\left(T N^{o}\right)$, this is equivalent to $\mathrm{pr}_{2} \circ \phi(X)=-\pi^{\sharp} \zeta$. Recall that $\sigma_{b}^{\prime}(X)=h^{*}\left(\sigma_{b}(X)-\phi^{*} \zeta\right)$.

Now, assume $\sigma=0$. Then $\sigma^{\prime}=0$ if, and only if $\sigma_{b}^{\prime}(X)=0$ for all $X \in T N$. From the previous discussion, this will be true if, and only if $h^{*}\left(\phi^{*} \zeta\right)=0$ for all $\zeta \in T N^{o}$ such that $\pi^{\sharp} \zeta \in \mathrm{pr}_{2} \circ \phi(T N)$, which is equivalent to

$$
T N^{o} \cap\left(\pi^{\sharp}\right)^{-1}\left(\mathrm{pr}_{2} \circ \phi(T N)\right) \subseteq\left(\phi^{*}\right)^{-1}\left(T N^{o}\right) .
$$

Let $A=\operatorname{pr}_{2} \circ \phi(T N)$. Now,

$$
\left(\pi^{\sharp}\right)^{-1}(A)=\left(\left(\pi^{\sharp}\right)^{*}\right)^{-1}(A)=\left(\pi^{\sharp}\left(A^{o}\right)\right)^{o},
$$

and

$$
\left(\phi^{*}\right)^{-1}\left(T N^{o}\right)=(\phi(T N))^{o} .
$$

Hence, (6-2) becomes

$$
\left(T N+\pi^{\sharp}\left(A^{o}\right)\right)^{o} \subseteq(\phi(T N))^{o},
$$

which is equivalent to

$$
\phi(T N) \subseteq T N+\pi^{\sharp}\left(A^{o}\right) .
$$

So a generalized complex submanifold $N$, of a generalized complex manifold $M$ with generalized complex structure (6-1), will have a generalized complex structure of the same form as (6-1) if and only if $\phi(T N) \subseteq T N+\pi^{\sharp}\left(A^{o}\right)$. Now, consider the following series of equivalent statements:

$$
\begin{aligned}
A & \subseteq \pi^{\sharp}\left(T N^{o}\right), \\
A^{o} & \supseteq\left(\pi^{\sharp}\left(T N^{o}\right)\right)^{o}, \\
A^{o} & \supseteq\left(\pi^{\sharp}\right)^{-1}(T N), \\
\pi^{\sharp}\left(A^{o}\right) & \supseteq \pi^{\sharp}\left(\left(\pi^{\sharp}\right)^{-1}(T N)\right)=T N .
\end{aligned}
$$

Thus (6-3) becomes

$$
\phi(T N) \subseteq \pi^{\sharp}\left(A^{o}\right) .
$$

If $N$ is both a complex submanifold of $(M, j)$, and a Poisson-Dirac submanifold of $(M, \pi)$ then this condition will automatically be satisfied and there will be a generalized complex structure of the form (6-1) on $N$. Thus $N$ will also be a holomorphic Poisson manifold. 


\section{Spinors and generalized complex submanifolds}

Generalized complex structures may also be realized using Clifford algebras and spinors. The aim of this section is to prove that generalized complex submanifolds can also be realized using spinors. Details for the material in this section can be found in [Gualtieri 2003] and the sections of [Chevalley 1997] cited therein.

Let $V$ be a finite dimensional vector space and let $\mathscr{C l}$ denote the Clifford algebra of $V_{\mathbb{C}} \oplus V_{\mathbb{C}}^{*}$. There is an action of $\mathscr{C} l$ on $\wedge^{\bullet}\left(V_{\mathbb{C}}^{*}\right)$ defined by

$$
(X+\xi) \cdot \mu=i_{X} \mu+\xi \wedge \mu,
$$

for all $X+\xi \in V_{\mathbb{C}} \oplus V_{\mathbb{C}}^{*}$ and $\mu \in \wedge^{\bullet}\left(V_{\mathbb{C}}^{*}\right)$. The elements of $\wedge^{\bullet}\left(V_{\mathbb{C}}^{*}\right)$ are called spinors. Each spinor $\mu$ has a null space:

$$
L_{\mu}=\left\{X+\xi \in V_{\mathbb{C}} \oplus V_{\mathbb{C}}^{*}:(X+\xi) \cdot \mu=0\right\} .
$$

This subspace is isotropic, and if it is also maximal isotropic then the spinor is called pure. Using the notation of Section 2, every pure spinor can be written as

$$
\mu=c\left(\operatorname{det}\left(R^{o}\right)\right) \wedge \exp (\varepsilon),
$$

where $c \in \mathbb{C}$ is nonzero, $R \subseteq V_{\mathbb{C}}$, and $\varepsilon \in \bigwedge^{2} R^{*}$. It is known that pure spinors, up to multiplication by a constant, are in one to one correspondence with maximal isotropics. The maximal isotropics $L_{\mu}$ and $\bar{L}_{\mu}=L_{\bar{\mu}}$ will have trivial intersection if, and only if,

$$
(\mu, \bar{\mu})_{\mathrm{muk}} \neq 0 .
$$

Here $(\cdot, \cdot)_{\text {muk }}$ is the Mukai pairing.

For each $m \in M$ the previous constructions can be applied to $\left(T_{m} M \oplus T_{m}^{*} M\right)^{\mathbb{C}}$. The bundle formed by these Clifford algebras is called the Clifford bundle. In this context spinors are members of $\Omega_{\mathbb{C}}^{\bullet}(M)$, and their null spaces are maximal isotropic subbundles of $T_{\mathbb{C}} M \oplus T_{\mathbb{C}}^{*} M$. The following proposition follows from [Alekseev and $\mathrm{Xu}$ 2001, Theorem 6.4]. It is also proven in [Gualtieri 2003] for the untwisted case.

Proposition 7.1. Generalized complex structures are in one to one correspondence with pure spinor line bundles $\mathscr{L} \subseteq \wedge^{\bullet} T_{\mathbb{C}}^{*} M$ such that the following hold.

(1) If $\mu \in \mathscr{L}$ then $(\mu, \bar{\mu})_{\mathrm{muk}} \neq 0$.

(2) For any local nonzero section $\mu \in \Gamma\left(\left.\mathscr{L}\right|_{U}\right)$ there exists a local section $X+\xi \in$ $\mathfrak{X}_{\mathbb{C}}(U) \oplus \Omega_{\mathbb{C}}(U)$ such that $d_{\Omega}(\mu)=(X+\xi) \cdot \mu$. Here $d_{\Omega}=d+\Omega$.

For each point $m \in M$ the spinor line $\left.\mathscr{L}\right|_{m}$ is of the form (7-1)

Let $h: N \rightarrow M$ be a twisted submanifold with a generalized complex structure defined by a spinor line bundle $\mathscr{L} \subseteq \wedge^{\bullet} T_{\mathbb{C}}^{*} M$. This spinor line bundle naturally induces a line bundle in $\Omega_{\mathbb{C}}^{\bullet}(N)$ given by $h^{*} \mathscr{L}$. This induced line bundle could 
potentially give a generalized complex structure on $N$. We will show that the maximal isotropic defined by $h^{*} \mathscr{L}$ is in fact $\Re_{h}(L \mathscr{L})$.

Proposition 7.2. Let $(M, J, \Omega)$ be a twisted generalized complex manifold and let $\theta$ be the pure spinor line that also gives $J$. If $h: N \rightarrow M$ is a twisted generalized complex submanifold of $M$, with generalized complex structure $J^{\prime}$, then the spinor associated to $J^{\prime}$ is $h^{*} \theta$.

Proof. Let $L$ denote the Dirac structure associated to $J$. The spinor line bundle associated to $L$ is given by

$$
\mathscr{L}=\left\{c\left(\operatorname{det}\left(R^{o}\right)\right) \wedge \exp (\varepsilon): c \in \mathbb{C}\right\} .
$$

Now,

$$
h^{*}\left(c \operatorname{det}\left(R^{o}\right) \wedge \exp (\varepsilon)\right)=c \operatorname{det}\left(\left(h^{-1} R\right)^{o}\right) \wedge \exp \left(h^{*} \varepsilon\right) .
$$

This line bundle is the same as the line bundle associated to $\mathscr{B}_{h}(L)$.

With this proposition it is now a simple matter to give the conditions for a twisted generalized complex submanifold in terms of spinors. The involutivity is guaranteed by Lemma 2.1 .

Corollary 7.3. Let $M$ be a twisted generalized complex submanifold, with associated spinor line bundle $\mathscr{L}$. A twisted submanifold $h: N \rightarrow M$ is a twisted generalized complex submanifold if, and only if, $h^{*} \mathscr{L}$ is a pure spinor line bundle and $\left(h^{*} \mu, h^{*} \bar{\mu}\right)_{\text {muk }} \neq 0$ for all $\mu \in \mathscr{L}$.

\section{Generalized Kähler submanifolds}

Finally we will consider submanifolds of generalized Kähler structures. A twisted generalized Kähler structure on $M$ is a pair of twisted generalized complex structures $J_{1}, J_{2}: T M \oplus T^{*} M \rightarrow T M \oplus T^{*} M$ such that

(1) $J_{1}$ and $J_{2}$ commute,

(2) $\left\langle X+\xi, J_{1} J_{2}(Y+\eta)\right\rangle$ is a positive definite metric.

The first proposition of this section gives a condition, in terms of the eigenbundles, for when two complex maps will commute.

Proposition 8.1. Let $W$ be a real vector space with two maps $\psi_{1}, \psi_{2}: W \rightarrow W$ such that $\psi_{1}^{2}=\psi_{2}^{2}=-\mathrm{Id}$. Also, let $L_{+}^{k}$ denote the $+i$-eigenbundles of these maps, and $L_{-}^{k}$ denote the $-i$-eigenbundles. Using this notation, $\psi_{1}$ and $\psi_{2}$ commute if, and only if

$$
W_{\mathbb{C}}=\left(L_{+}^{1} \cap L_{+}^{2}\right) \oplus\left(L_{+}^{1} \cap L_{-}^{2}\right) \oplus\left(L_{-}^{1} \cap L_{+}^{2}\right) \oplus\left(L_{-}^{1} \cap L_{-}^{2}\right) .
$$


Proof. First assume the two maps commute. Because of this fact, every $w \in W_{\mathbb{C}}$ can be written as

$$
\begin{aligned}
w= & \frac{1}{4}\left(\operatorname{Id}-i J_{1}\right)\left(\operatorname{Id}-i J_{2}\right)(w)+\frac{1}{4}\left(\operatorname{Id}-i J_{1}\right)\left(\operatorname{Id}+i J_{2}\right)(w) \\
& +\frac{1}{4}\left(\operatorname{Id}+i J_{1}\right)\left(\operatorname{Id}-i J_{2}\right)(w)+\frac{1}{4}\left(\operatorname{Id}+i J_{1}\right)\left(\operatorname{Id}+i J_{2}\right)(w) \\
:= & w_{+}^{+}+w_{-}^{+}+w_{+}^{-}+w_{-}^{-} .
\end{aligned}
$$

It is clear that $w_{\bullet}^{ \pm} \in L_{ \pm}^{1}$, and $w_{ \pm}^{\bullet} \in L_{ \pm}^{2}$. Now assume every $w \in W_{\mathbb{C}}$ can be written as $w=w_{+}^{+}+w_{-}^{+}+w_{+}^{-}+w_{-}^{-}$, where $w_{\bullet}^{ \pm} \in L_{ \pm}^{1}$ and $w_{ \pm}^{\bullet} \in L_{ \pm}^{2}$. Now $\left(\psi_{2} \circ \psi_{1}\right)(w)=\psi_{2}\left(i w_{+}^{+}+i w_{-}^{+}-i w_{+}^{-}-i w_{+}^{-}\right)=-w_{-}^{+}+w_{-}^{+}+w_{+}^{-}-w_{-}^{-}$, and $\left(\psi_{1} \circ \psi_{2}\right)(w)=-w_{+}^{+}+w_{-}^{+}+w_{+}^{-}-w_{-}^{-}$.

Let $J_{1}, J_{2}: T M \oplus T^{*} M \rightarrow T M \oplus T^{*} M$ be two commuting bundle maps such that $J_{1}^{2}=J_{2}^{2}=-$ Id. Also, let $L_{ \pm}^{k}$ denote the $+i$-eigenbundles and $-i$-eigenbundles of $J_{k}$. We also use the notation of Section 4 , in which $B=\left.T N \oplus T^{*} M\right|_{N}$, and $s: B \rightarrow T N \oplus T^{*} N$. The next two lemmas relate the condition above to our conditions.

Lemma 8.2. The following are equivalent.

(1) $T_{\mathbb{C}} N \oplus T_{\mathbb{C}}^{*} N=s\left(\left(L_{+}^{1} \cap L_{+}^{2}\right) \cap B_{\mathbb{C}}\right)+s\left(\left(L_{+}^{1} \cap L_{-}^{2}\right) \cap B_{\mathbb{C}}\right)+s\left(\left(L_{-}^{1} \cap L_{+}^{2}\right) \cap\right.$ $\left.B_{\mathbb{C}}\right)+s\left(\left(L_{-}^{1} \cap L_{-}^{2}\right) \cap B_{\mathbb{C}}\right)$.

(2) $T N \oplus T^{*} N=s\left(B \cap J_{1} B \cap J_{2} B \cap J_{1} J_{2} B\right)$.

(3) $B=B \cap J_{1} B \cap J_{2} B \cap J_{1} J_{2} B+B^{\perp}$.

(4) $J_{1} B^{\perp} \cap B \subseteq B^{\perp}, J_{2} B^{\perp} \cap B \subseteq B^{\perp}$, and $B \cap J_{1} J_{2} B^{\perp} \subseteq B^{\perp}$.

Proof. (1) $\Longrightarrow(2)$ : Every $v \in T_{\mathbb{C}} N \oplus T_{\mathbb{C}}^{*} N$ can be written as $v=s\left(\tilde{v}_{+}^{+}\right)+s\left(\tilde{v}_{-}^{+}\right)+$ $s\left(\tilde{v}_{+}^{-}\right)+s\left(\tilde{v}_{-}^{-}\right)$, where $\tilde{v}_{ \pm}^{+} \in L_{+}^{1} \cap L_{ \pm}^{2} \cap B_{\mathbb{C}}$ and $\tilde{v}_{ \pm}^{-} \in L_{-}^{1} \cap L_{ \pm}^{2} \cap B_{\mathbb{C}}$. Now let $\tilde{v}=\tilde{v}_{+}^{+}+\tilde{v}_{-}^{+}+\tilde{v}_{+}^{-}+\tilde{v}_{-}^{-}$, and so $v=s(\tilde{v})$ and $\tilde{v} \in B_{\mathbb{C}}$. Now $J_{1}(\tilde{v}) \in B_{\mathbb{C}}, J_{2}(\tilde{v}) \in B_{\mathbb{C}}$, and $J_{1} J_{2}(\tilde{v}) \in B_{\mathbb{C}}$. Thus $\tilde{v} \in J_{1}\left(B_{\mathbb{C}}\right), \tilde{v} \in J_{2}\left(B_{\mathbb{C}}\right)$, and $\tilde{v} \in J_{1} J_{2}\left(B_{\mathbb{C}}\right)$. Finally, taking the real parts of each of these gives (2).

(2) $\Longrightarrow(1)$ : Every $v \in T N \oplus T^{*} N$ can be written as $v=s(\tilde{v})$ for some $\tilde{v} \in$ $B \cap J_{1} B \cap J_{2} B \cap J_{1} J_{2} B$. Alternately $v=s(\tilde{v})$ for some $\tilde{v} \in B$ such that $J_{1}(\tilde{v}) \in B$, $J_{2}(\tilde{v}) \in B$, and $J_{1} J_{2}(\tilde{v}) \in B$. Now we can write

$$
\begin{aligned}
\tilde{v}=\frac{1}{4}\left(\left(\operatorname{Id}-i J_{1}\right) \circ\left(\operatorname{Id}-i J_{2}\right)(\tilde{v})+\left(\operatorname{Id}-i J_{1}\right) \circ\left(\operatorname{Id}+i J_{2}\right)(\tilde{v})\right. \\
\left.+\left(\operatorname{Id}+i J_{1}\right) \circ\left(\operatorname{Id}-J_{2}\right)(\tilde{v})+\left(\operatorname{Id}+i J_{1}\right) \circ\left(\operatorname{Id}+i J_{2}\right)(\tilde{v})\right) .
\end{aligned}
$$

By definition each of these components is in the intersection of the eigenbundles, and the previous discussion shows that each of these terms is also in $B_{\mathbb{C}}$.

(2) $\Longleftrightarrow$ (3): We know $s(B)=T_{\mathbb{C}} N \oplus T_{\mathbb{C}}^{*} N$, and $\operatorname{ker}(s)=B^{\perp}$. Thus these two conditions are equivalent. 
(3) $\Longleftrightarrow(4)$ : This equivalence is a fairly straightforward calculation:

$$
B=B^{\perp}+B \cap J_{1} B \cap J_{2} B \cap J_{1} J_{2} B,
$$

if, and only if

$$
B \subseteq B^{\perp}+J_{1} B \cap J_{2} B \cap J_{1} J_{2} B
$$

if, and only if

$$
B \cap\left(J_{1} B^{\perp}+J_{2} B^{\perp}+J_{1} J_{2} B^{\perp}\right) \subseteq B^{\perp},
$$

if, and only if

$$
B \cap J_{1} B^{\perp}+B \cap J_{2} B^{\perp}+B \cap J_{1} J_{2} B^{\perp} \subseteq B^{\perp},
$$

if, and only if

$$
B \cap J_{1} B^{\perp} \subseteq B^{\perp}, B \cap J_{2} B^{\perp} \subseteq B^{\perp} \text {, and } B \cap J_{1} J_{2} B^{\perp} \subseteq B^{\perp} .
$$

This last lemma strengthens the conclusions of the first statement in Lemma 8.2.

Lemma 8.3. If $N$ is a twisted generalized complex submanifold of $\left(M, J_{1}\right)$ and $\left(M, J_{2}\right)$ then the sums in expression (1), of the previous proposition, are direct. Also, each of the components in this expression can be rewritten as

$$
s\left(\left(L_{ \pm}^{1} \cap L_{ \pm}^{2}\right) \cap B_{\mathbb{C}}\right)=F_{ \pm}^{1} \cap F_{ \pm}^{2},
$$

where $F_{ \pm}^{k}=\mathscr{B}_{i}\left(L_{ \pm}^{k}\right)$.

Proof. The fact that $J_{1}$ and $J_{2}$ descend to generalized complex structures on $N$ implies that $F_{+}^{k} \cap F_{-}^{k}=\{0\}$, and the sums must be direct. Now, by definition $s\left(L_{ \pm}^{k} \cap B_{\mathbb{C}}\right)=F_{ \pm}^{k}$ and it is obvious that $s\left(\left(L_{ \pm}^{1} \cap L_{ \pm}^{2}\right) \cap B_{\mathbb{C}}\right) \subseteq F_{ \pm}^{1} \cap F_{ \pm}^{2}$. To see the other inclusion, consider $F_{+}^{1} \cap F_{+}^{2}$. This subset will have zero intersection with $F_{-}^{1}$ and $F_{-}^{2}$, and so it will not intersect with any of the other components. However, $F_{+}^{1} \cap F_{+}^{2} \subseteq T_{\mathbb{C}} N \oplus T_{\mathbb{C}}^{*} N$, and the fact that $T_{\mathbb{C}} N \oplus T_{\mathbb{C}}^{*} N$ is made up of these four components implies that $F_{+}^{1} \cap F_{+}^{2} \subseteq s\left(\left(L_{ \pm}^{1} \cap L_{ \pm}^{2}\right) \cap B_{\mathbb{C}}\right)$.

We are now ready to prove our last theorem, namely that these conditions are guaranteed to be satisfied by a generalized Kähler structure and so our notion of generalized complex submanifold preserves generalized Kähler structures.

Theorem 8.4. Let $N$ be a twisted submanifold of a generalized Kähler submanifold $\left(M, J_{1}, J_{2}\right)$. If $N$ is a twisted generalized complex submanifold of $\left(M, J_{1}\right)$ and $\left(M, J_{2}\right)$, then $\left(N, J_{1}^{\prime}, J_{2}^{\prime}\right)$ is automatically a twisted generalized Kähler manifold.

Proof. All that we need to show is $J_{1}^{\prime} J_{2}^{\prime}=J_{2}^{\prime} J_{1}^{\prime}$, and the metric induced by $G^{\prime}=$ $J_{1}^{\prime} J_{2}^{\prime}$ is positive definite. We start with the commutativity. By Lemma 8.3 and Proposition 8.1, if one of the equivalent conditions in Lemma 8.2 is true then $J_{1}$ and $J_{2}$ will commute. Consider condition (4) of this lemma. By assumption $J_{1} B^{\perp} \cap B \subseteq B^{\perp}$ and $J_{2} B^{\perp} \cap B \subseteq B^{\perp}$. All that remains is to show $B \cap J_{1} J_{2} B^{\perp} \subseteq B^{\perp}$. 
Take $v \in B \cap J_{1} J_{2} B^{\perp}$, so $v \in B^{\perp}$ and $J_{1} J_{2} v \in B$. Thus $\left\langle v, J_{1} J_{2} v\right\rangle=0$. However, by assumption this metric is positive definite and so $v=0$. Thus $B \cap J_{1} J_{2} B^{\perp} \subseteq\{0\}$, and $B \cap J_{1} J_{2} B^{\perp} \subseteq B^{\perp}$ is always true. It remains to show that $J_{1}^{\prime} J_{2}^{\prime}$ defines a positive definite metric. Take $v \in T M \oplus T^{*} M$ and $\tilde{v} \in B \cap J_{1} B \cap J_{2} B \cap J_{1} J_{2} B$ such that $s(\tilde{v})=v$. Because $s$ does not change the inner product

$$
\left\langle v, J_{1}^{\prime} J_{2}^{\prime}(v)\right\rangle=\left\langle s(\tilde{v}), J_{1}^{\prime} J_{2}^{\prime} s(\tilde{v})\right\rangle=\left\langle s(\tilde{v}), s J_{1} J_{2}(\tilde{v})\right\rangle=\left\langle\tilde{v}, J_{1} J_{2}(\tilde{v})\right\rangle,
$$

and the positive definiteness of $J_{1} J_{2}$ implies the positive definiteness of $J_{1}^{\prime} J_{2}^{\prime}$.

\section{Acknowledgements}

We would like to thank Izu Vaisman for pointing out an error in an earlier version of this paper.

\section{References}

[Abouzaid and Boyarchenko 2006] M. Abouzaid and M. Boyarchenko, "Local structure of generalized complex manifolds", J. Symplectic Geom. 4:1 (2006), 43-62. MR 2007e:53106 Zbl 1116. 53055

[Alekseev and Xu 2001] A. Alekseev and P. Xu, "Derived brackets and courant algebroids", unpublished manuscript, 2001.

[Barton 2007] J. Barton, Generalized complex structures on Courant algebroids, Ph.D. thesis, The Pennsylvania State University, 2007.

[Ben-Bassat and Boyarchenko 2004] O. Ben-Bassat and M. Boyarchenko, "Submanifolds of generalized complex manifolds", J. Symplectic Geom. 2:3 (2004), 309-355. MR 2006b:53067 Zbl 1082.53077

[Bursztyn and Radko 2003] H. Bursztyn and O. Radko, "Gauge equivalence of Dirac structures and symplectic groupoids”, Ann. Inst. Fourier (Grenoble) 53:1 (2003), 309-337. MR 2004d:53101 Zbl 1026.58019

[Chevalley 1997] C. Chevalley, The algebraic theory of spinors and Clifford algebras, vol. 2 in his Collected Works, Springer, Berlin, 1997. MR 99f:01028 Zbl 0899.01032

[Courant 1990] T. J. Courant, "Dirac manifolds", Trans. Amer. Math. Soc. 319:2 (1990), 631-661. MR 90m:58065 Zbl 0850.70212

[Crainic 2004] M. Crainic, "Generalized complex structures and Lie brackets", preprint, 2004. arXiv math/0412097

[Crainic and Fernandes 2004] M. Crainic and R. L. Fernandes, "Integrability of Poisson brackets", J. Differential Geom. 66:1 (2004), 71-137. MR 2006f:53119 Zbl 1066.53131

[Fernandes and Vanhaecke 2001] R. L. Fernandes and P. Vanhaecke, "Hyperelliptic Prym varieties and integrable systems", Comm. Math. Phys. 221:1 (2001), 169-196. MR 2002g:37072 Zbl 0991.37038

[Gualtieri 2003] M. Gualtieri, Generalized complex geometry, Ph.D. thesis, Oxford University, 2003. arXiv math/0401221

[Gualtieri 2007] M. Gualtieri, "Generalized complex geometry”, preprint, 2007. arXiv math.DG/ 0703298. 
[Hitchin 2003] N. Hitchin, "Generalized Calabi-Yau manifolds", Q. J. Math. 54:3 (2003), 281-308. MR 2004h:32024 Zbl 1076.32019

[Hu 2005] S. Hu, "Hamiltonian symmetries and reduction in generalized geometry", preprint, 2005. arXiv math/0509060

[Lindström et al. 2005] U. Lindström, R. Minasian, A. Tomasiello, and M. Zabzine, "Generalized complex manifolds and supersymmetry", Comm. Math. Phys. 257:1 (2005), 235-256. MR 2006f: 53111 Zbl 1118.53048

[Liu et al. 1997] Z.-J. Liu, A. Weinstein, and P. Xu, "Manin triples for Lie bialgebroids", J. Differential Geom. 45:3 (1997), 547-574. MR 98f:58203 Zbl 0885.58030

[Mackenzie and Xu 1994] K. C. H. Mackenzie and P. Xu, "Lie bialgebroids and Poisson groupoids", Duke Math. J. 73:2 (1994), 415-452. MR 95b:58171 Zbl 0844.22005

[Roytenberg 1999] D. Roytenberg, Courant algebroids, derived brackets and even symplectic supermanifolds, Ph.D. thesis, Univ. California Berkeley, 1999. arXiv math/9910078

[Ševera and Weinstein 2001] P. Ševera and A. Weinstein, "Poisson geometry with a 3-form background”, Progr. Theoret. Phys. Suppl. 144 (2001), 145-154. MR 2005e:53132 Zbl 1029.53090

[Stiénon and Xu 2007] M. Stiénon and P. Xu, "Poisson quasi-Nijenhuis manifolds", Comm. Math. Phys. 270:3 (2007), 709-725. MR 2007k:53136 Zbl 1119.53056

[Stiénon and Xu 2008] M. Stiénon and P. Xu, "Reduction of generalized complex structures", $J$. Geom. Phys. 58:1 (2008), 105-121.

[Vaisman 2007] I. Vaisman, "Reduction and submanifolds of generalized complex manifolds", Differential Geom. Appl. 25:2 (2007), 147-166. MR 2008b:53112 Zbl 1126.53049

[Xu 2003] P. Xu, "Dirac submanifolds and Poisson involutions", Ann. Sci. École Norm. Sup. (4) 36:3 (2003), 403-430. MR 2004e:53125 Zbl 1047.53052

Received August 12, 2007. Revised January 21, 2008.

\author{
JAMES BARTON \\ DEPARTMENT OF MATHEMATICS \\ PENNSYlVANia State UniVERSity \\ 109 MCALLISTER BUILDING \\ UNIVERSITY PARK, PA 16802 \\ UNITED STATES \\ barton@math.psu.edu \\ MATHieU StiÉNON \\ ETH ZÜRICH \\ DEPARTEMENT MATHEMATIK \\ RÄMISTRASSE 101 \\ 8092 ZÜRICH \\ SWITZERLAND \\ stienon@math.ethz.ch
}

